PROCEEDINGS OF THE

AMERICAN MATHEMATICAL SOCIETY

Volume 132, Number 8, Pages 2265-2271

S 0002-9939(04)07526-4

Article electronically published on March 25, 2004

\title{
A GEOMETRICAL VERSION OF HARDY'S INEQUALITY FOR $\stackrel{\circ}{\mathrm{W}}^{1, p}(\Omega)$
}

\author{
JESPER TIDBLOM
}

(Communicated by Jonathan M. Borwein)

\begin{abstract}
The aim of this article is to prove a Hardy-type inequality, concerning functions in $\stackrel{\circ}{\mathrm{W}}^{1, p}(\Omega)$ for some domain $\Omega \subset R^{n}$, involving the volume of $\Omega$ and the distance to the boundary of $\Omega$. The inequality is a generalization of a recently proved inequality by M. Hoffmann-Ostenhof, T. Hoffmann-Ostenhof and A. Laptev (2002), which dealt with the special case $p=2$.
\end{abstract}

\section{INTRODUCTION}

The history of Hardy-type inequalities goes back to Hardy and the 1920s when the following original one-dimensional inequality appeared in $[8]$ :

$$
\int_{0}^{\infty}\left(\frac{F(x)}{x}\right)^{p} d x \leq\left(\frac{p}{p-1}\right)^{p} \int_{0}^{\infty} f(x)^{p} d x
$$

where

$$
p>1, \quad f(x) \geq 0 \text { and } F(x)=\int_{0}^{x} f(t) d t
$$

(see also 7]). A multidimensional version of this inequality is

$$
\int_{R^{n}}|\nabla u|^{p} d x \geq\left|\frac{n-p}{p}\right|^{p} \int_{R^{n}} \frac{|u(x)|^{p}}{|x|^{p}} d x, \quad u \in C_{0}^{\infty}\left(\mathbb{R}^{n} \backslash\{0\}\right),
$$

where $p>1$ and the constant $\left|\frac{n-p}{p}\right|^{p}$ is optimal (see for example [13]).

Later on, these inequalities have been generalized and modified in many different ways, and the literature concerning such inequalities is extensive. There is an entire book by B. Opic and A. Kufner devoted to various Hardy-type inequalities (see [13). Many other Hardy-Sobolev type inequalities may be found in the excellent book "Sobolev Spaces" 12 by V. G. Maz'ja.

In the past few years, many articles on the subject have been published; see [3] for a review of recent results in the field. In the article [1, G. Barbatis, S. Filippas and A. Tertikas give a very comprehensive treatment of improved $L^{p}$ Hardy inequalities with best constants, involving various kinds of distance functions.

Received by the editors January 28, 2002.

2000 Mathematics Subject Classification. Primary 35P99; Secondary 35P20, 47A75, 47B25.

(C)2004 American Mathematical Society 
Let $\Omega$ be an open set in $\mathbb{R}^{n}$ and $\delta(x)=\operatorname{dist}(x, \partial \Omega)$. It is known (see, for example, 11]) that for any $p>1$ we have

$$
\int_{\Omega}|\nabla u|^{p} d x \geq c_{p} \int_{\Omega} \frac{|u|^{p}}{\delta(x)^{p}} d x, \quad u \in \stackrel{\circ}{\mathrm{W}}^{1, p}(\Omega),
$$

where $\Omega$ is convex and $c_{p}=\left(\frac{p-1}{p}\right)^{p}$ is the best constant (see, for example, [1] ). $\stackrel{\circ}{\mathrm{W}}^{1, p}(\Omega)$ as usual is the completion of $C_{0}^{\infty}(\Omega)$ with respect to the Sobolev norm

$$
\|u\|_{\mathrm{W}^{1, p}(\Omega)}=\|u\|_{L^{p}(\Omega)}+\sum_{i=1}^{n}\left\|\frac{\partial u}{\partial x_{i}}\right\|_{L^{p}(\Omega)} .
$$

The main result to be proved in this paper is that an extra term of the form

$$
\frac{a(p, n)}{|\Omega|^{\frac{p}{n}}} \int_{\Omega}|u(x)|^{p} d x \quad(a(p, n)>0),
$$

where $|\Omega|=\operatorname{vol}(\Omega)$, may be added to the right-hand side of the inequality (0.1).

Let $\Omega \subset \mathbb{R}^{n}$ be an arbitrary convex domain. In [2], H. Brezis and M. Marcus proved that the largest possible constant $\lambda(\Omega)$ in the inequality

$$
\int_{\Omega}|\nabla u(x)|^{2} d x \geq \frac{1}{4} \int_{\Omega} \frac{|u(x)|^{2}}{\delta^{2}(x)} d x+\lambda(\Omega) \int_{\Omega}|u(x)|^{2} d x, \quad u \in \stackrel{\circ}{\mathrm{W}}{ }^{1,2}(\Omega),
$$

satisfies

$$
\lambda(\Omega) \geq \frac{1}{4 \cdot \operatorname{diam}^{2}(\Omega)}
$$

In the same paper, H. Brezis and M. Marcus have asked whether the above estimate can be replaced by some other estimate of the type $\lambda(\Omega) \geq \alpha|\Omega|^{-2 / n}$ for some universal constant $\alpha>0$.

This question was recently answered in the affirmative by M. Hoffmann-Ostenhof, T. Hoffmann-Ostenhof and A. Laptev in [9]. In that paper, the following Hardytype inequality

$$
\int_{\Omega}|\nabla u(x)|^{2} d x \geq \frac{1}{4} \int_{\Omega} \frac{|u(x)|^{2}}{\delta^{2}(x)} d x+\frac{\mu_{n}}{|\Omega|^{\frac{2}{n}}} \int_{\Omega}|u(x)|^{2} d x, \quad u \in \stackrel{\circ}{\mathrm{W}^{1,2}}(\Omega),
$$

where

is established.

$$
\mu_{n}=\frac{n^{(n-2) / n}\left|\mathbb{S}^{n-1}\right|^{2 / n}}{4}
$$

Here we shall prove a similar "geometric" inequality for functions from the Sobolev space $\stackrel{\circ}{\mathrm{W}}^{1, p}(\Omega)$. More precisely, we shall prove

$$
\int_{\Omega}|\nabla u|^{p} d x \geq c_{p} \int_{\Omega} \frac{|u(x)|^{p}}{\delta^{p}(x)} d x+\frac{a(p, n)}{|\Omega|^{\frac{p}{n}}} \int_{\Omega}|u(x)|^{p} d x, \quad u \in \stackrel{\circ}{\mathrm{W}}^{1, p}(\Omega),
$$

where

$$
a(p, n)=\frac{(p-1)^{p+1}}{p^{p}} \cdot\left(\frac{\left|\mathbb{S}^{n-1}\right|}{n}\right)^{\frac{p}{n}} \cdot \frac{\sqrt{\pi} \cdot \Gamma\left(\frac{n+p}{2}\right)}{\Gamma\left(\frac{p+1}{2}\right) \Gamma\left(\frac{n}{2}\right)} .
$$

The latter inequality is a generalization of inequality $(0.2)$ for any $p>1$. In particular, $a(2, n)=\mu_{n}$.

In section one, we shall, following a method from [9], prove a one-dimensional version of the inequality and in section two, we shall extend it to higher dimensions. 


\section{One-Dimensional INEQualities}

Let $f$ be a function defined and differentiable on $(0, b]$ for some $b>0$. We say that $f$ belongs to the class $\Phi_{p}(0, b)$ if $f$ is real-valued and there exists a constant $C=C(f)$ such that

$$
\sup _{0<t \leq b}\left(t^{p-1}|f(t)|+t^{p}\left|f^{\prime}(t)\right|\right) \leq C .
$$

Throughout this article it is assumed that $p>1$.

Lemma 1.1. Let $u \in C^{1}[0, b], b>0, u(0)=0$ and let $f \in \Phi_{p}(0, b)$. Then we have the following inequality:

$$
\int_{0}^{b}\left|u^{\prime}(t)\right|^{p} d t \geq \frac{1}{p^{p}} \frac{\left.\left.\left|\int_{0}^{b} f^{\prime}(t)\right| u(t)\right|^{p} d t\right|^{p}}{\left(\int_{0}^{b}|f(t)-f(b)|^{\frac{p}{p-1}}|u(t)|^{p} d t\right)^{p-1}} .
$$

Proof. Let $c$ be a constant. We have

$$
\begin{aligned}
\left.|(f(b)-c)| u(b)\right|^{p} & -\int_{0}^{b} f^{\prime}(t)|u(t)|^{p} d t \mid \\
& =\left|\int_{0}^{b}(f(t)-c)\left(|u(t)|^{p}\right)^{\prime} d t\right| \\
& =\frac{p}{2}\left|\int_{0}^{b}(f(t)-c)\left(u^{\frac{p}{2}-1} \bar{u}^{\frac{p}{2}} u^{\prime}+\bar{u}^{\frac{p}{2}-1} u^{\frac{p}{2}} \bar{u}^{\prime}\right) d t\right| \\
& \leq p \int_{0}^{b}|f(t)-c||u|^{p-1}\left|u^{\prime}\right| d t \\
& \leq p\left(\int_{0}^{b}|f(t)-c|^{\frac{p}{p-1}}|u|^{p} d t\right)^{\frac{p-1}{p}}\left(\int_{0}^{b}\left|u^{\prime}\right|^{p} d t\right)^{\frac{1}{p}} .
\end{aligned}
$$

Now put $c=f(b)$ and raise both sides to the power $p$. We get

$$
\left.\left.\left|\int_{0}^{b} f^{\prime}(t)\right| u\right|^{p} d t\right|^{p} \leq p^{p}\left(\int_{0}^{b}|f(t)-f(b)|^{\frac{p}{p-1}}|u|^{p} d t\right)^{p-1} \int_{0}^{b}\left|u^{\prime}\right|^{p} d t
$$

and we are done.

Corollary 1.1. Let $u$ be as in the lemma above, and put $f(t)=\frac{t^{1-p}}{1-p} \in \Phi_{p}(0, b)$. Then the following improved Hardy inequality holds:

$$
\begin{aligned}
\int_{0}^{b}\left|u^{\prime}(t)\right|^{p} d t & \geq c_{p} \frac{\left(\int_{0}^{b} \frac{|u|^{p}}{t^{p}} d t\right)^{p}}{\left(\left.\int_{0}^{b}\left|t^{1-p}-b^{1-\left.p\right|^{\frac{p}{p-1}}}\right| u\right|^{p} d t\right)^{p-1}} \\
& \geq c_{p} \int_{0}^{b} \frac{|u|^{p}}{t^{p}} d t .
\end{aligned}
$$

Proof. Use the previous lemma. 
Now we give a linearized version of the corollary:

Corollary 1.2 (linearized version). Let $u$ be as above. Then

$$
\int_{0}^{b}\left|u^{\prime}(t)\right|^{p} d t \geq c_{p}\left(\int_{0}^{b}\left(\frac{p}{t^{p}}-(p-1)\left(t^{1-p}-b^{1-p}\right)^{\frac{p}{p-1}}\right)|u|^{p} d t\right) .
$$

Proof. Young's inequality gives us

$$
\frac{A^{p}}{B^{p-1}} \geq p A-(p-1) B .
$$

If we put $A=\int_{0}^{b} \frac{|u|^{p}}{t^{p}} d t, B=\int_{0}^{b}\left|t^{1-p}-b^{1-p}\right|^{\frac{p}{p-1}}|u|^{p} d t$ and use Corollary 1.1, we get (1.1).

An easy consequence of Corollary 1.2 (see also [9]) is

Lemma 1.2. Let $u \in \stackrel{\circ}{\mathrm{W}}^{1, p}(0,2 b), b>0$. Then we have

$$
\int_{0}^{2 b}\left|u^{\prime}(t)\right|^{p} d t \geq c_{p} \int_{0}^{2 b}\left(\frac{p}{\rho(t)^{p}}-(p-1)\left(\frac{1}{\rho(t)^{p-1}}-\frac{1}{b^{p-1}}\right)^{\frac{p}{p-1}}\right)|u(t)|^{p} d t,
$$

where

$$
\rho(t)=\operatorname{dist}(t, \mathbb{R} \backslash[0,2 b])=\min (t, 2 b-t) .
$$

Proof. By rewriting the inequality (1.1) for the interval $[b, 2 b]$ for functions $u \in$ $C^{1}[b, 2 b]$ such that $u(2 b)=0$, we get

$$
\int_{b}^{2 b}\left|u^{\prime}(t)\right|^{p} d t \geq c_{p} \int_{b}^{2 b}\left(\frac{p}{(2 b-t)^{p}}-\frac{(p-1)}{\left((2 b-t)^{1-p}-b^{1-p}\right)^{\frac{-p}{p-1}}}\right)|u|^{p} d t .
$$

If we add the inequalities (1.1) and (1.2) and use standard density arguments, we get the statement of the lemma.

Theorem 1.1 (one-dimensional version). Let $u \in \stackrel{\circ}{\mathrm{W}}^{1, p}(a, b)$. Then we have

$$
\int_{a}^{b}\left|u^{\prime}(t)\right|^{p} d t \geq c_{p}\left(\int_{a}^{b} \frac{|u(t)|^{p}}{\rho(t)^{p}} d t+\frac{p-1}{\left(\frac{b-a}{2}\right)^{p}} \int_{a}^{b}|u(t)|^{p} d t\right) .
$$

Proof. Without loss of generality, we can assume that the interval of integration is $[0,2 b]$. The right-hand side in Lemma 1.2 may be written

$$
c_{p}\left(\int_{0}^{2 b} \frac{|u(t)|^{p}}{\rho(t)^{p}} d t+\int_{0}^{2 b} \frac{p-1}{\rho(t)^{p}}\left(1-\left(1-\left(\frac{\rho(t)}{b}\right)^{p-1}\right)^{\frac{p}{p-1}}\right)|u(t)|^{p} d t\right) .
$$

We will now estimate the expression in front of $|u|^{p}$ in the last integral from below. We begin by noticing that $\rho(t) \leq b$. We get

$$
\begin{aligned}
\frac{1}{\rho(t)^{p}}\left(1-\left(1-\left(\frac{\rho(t)}{b}\right)^{p-1}\right)^{\frac{p}{p-1}}\right) & \geq \frac{1}{\rho(t)^{p}}\left(1-\left(1-\left(\frac{\rho(t)}{b}\right)^{p-1}\right)\right) \\
& =\frac{1}{\rho(t) b^{p-1}} \geq \frac{1}{b^{p}} .
\end{aligned}
$$

This, together with Lemma 1.2, immediately gives us inequality (1.3). 


\section{INEQUALITIES IN HIGHER DIMENSIONS}

In this section we will extend the one-dimensional results in the previous section to higher dimensions, using almost the same arguments as in [9]. For simplicity I use the same notation as in the mentioned article. If $\nu \in \mathbb{S}^{n-1}$, we put

$$
\begin{gathered}
\tau_{\nu}(x)=\min \{s>0: x+s \nu \notin \Omega\}, \quad \rho_{\nu}(x)=\min \left(\tau_{\nu}(x), \tau_{-\nu}(x)\right), \\
D_{\nu}(x)=\tau_{\nu}(x)+\tau_{-\nu}(x), \quad \Omega_{x}=\{y \in \Omega: x+t(y-x) \in \Omega, \forall t \in[0,1]\}, \\
\delta(x)=\inf _{\nu \in S^{n-1}} \tau_{\nu}(x)=\operatorname{dist}(x, \partial \Omega),
\end{gathered}
$$

where $d \omega(\nu)$ denotes the normalized surface measure on $\mathbb{S}^{n-1}, \int_{S^{n-1}} d \omega(\nu)=1$.

Before stating our main theorem, we need an auxiliary lemma.

\section{Lemma 2.1.}

$$
\int_{S^{n-1}}\left(\frac{2}{D_{\nu}(x)}\right)^{p} d \omega(\nu) \geq\left(\frac{n\left|\Omega_{x}\right|}{\left|\mathbb{S}^{n-1}\right|}\right)^{-\frac{p}{n}}
$$

Proof. Since the function $f(t)=t^{-p}$ is convex when $t>0$, we can use Jensen's inequality to get

$$
\int_{S^{n-1}}\left(\frac{2}{D_{\nu}(x)}\right)^{p} d \omega(\nu) \geq\left(\int_{S^{n-1}}\left(\frac{D_{\nu}(x)}{2}\right) d \omega(\nu)\right)^{-p}
$$

Since

$$
\begin{aligned}
\int_{S^{n-1}}\left(\frac{D_{\nu}(x)}{2}\right) d \omega(\nu) & =\frac{1}{2} \int_{S^{n-1}} \tau_{\nu}+\tau_{-\nu} d \omega(\nu) \\
& =\int_{S^{n-1}} \tau_{\nu} d \omega(\nu) \\
& \leq\left(\int_{S^{n-1}} \tau_{\nu}^{n} d \omega(\nu)\right)^{\frac{1}{n}} \\
& =\left(\frac{n\left|\Omega_{x}\right|}{\left|\mathbb{S}^{n-1}\right|}\right)^{\frac{1}{n}}
\end{aligned}
$$

we obtain 2.1.

We are now ready for the main theorem.

Theorem 2.1. Let $\Omega$ be a domain in $\mathbb{R}^{n}$. Then the following Hardy-type inequality holds for all $u \in \stackrel{\circ}{\mathrm{W}}^{1, p}(\Omega), p>1$ :

$$
\begin{aligned}
\int_{\Omega}|\nabla u|^{p} d x & \geq \frac{c_{p} \sqrt{\pi} \cdot \Gamma\left(\frac{n+p}{2}\right)}{\Gamma\left(\frac{p+1}{2}\right) \Gamma\left(\frac{n}{2}\right)}\left(\int_{\Omega} \int_{S^{n-1}} \frac{1}{\rho_{v}(x)^{p}} d \omega(\nu)|u(x)|^{p} d x\right. \\
& \left.+(p-1)\left(\frac{\left|\mathbb{S}^{n-1}\right|}{n}\right)^{\frac{p}{n}} \int_{\Omega} \frac{|u(x)|^{p}}{\left|\Omega_{x}\right|^{\frac{p}{n}}} d x\right)
\end{aligned}
$$

Proof. Clearly, we can assume $u \in C_{0}^{\infty}(\Omega)$. At first, we also assume that $u$ is real valued. E. B. Davies arguments (see [6]) together with the one-dimensional inequality (Theorem 1.1) give

$$
\int_{\Omega}\left|\partial_{\nu} u\right|^{p} d x \geq c_{p} \int_{\Omega} \frac{|u(x)|^{p}}{\rho_{\nu}(x)^{p}} d x+c_{p}(p-1) \int_{\Omega}\left(\frac{2}{D_{\nu}(x)}\right)^{p}|u(x)|^{p} d x .
$$


By definition, we have

$$
\left|\partial_{\nu} u\right|=|\nu \cdot \nabla u|=|\nabla u||\cos (\nu, \nabla u)|,
$$

where $\cos (v, w)$ denotes the angle between $v, w \in \mathbb{R}^{n}$. By inserting this into the above inequality and integrating both sides with respect to the normalized surface measure on $\mathbb{S}^{n-1}$, we get

$$
\begin{gathered}
\int_{\Omega} \int_{S^{n-1}}|\cos (\nu, \nabla u)|^{p} d \omega(\nu)|\nabla u(x)|^{p} d x \\
\geq c_{p}\left(\int_{\Omega} \int_{S^{n-1}}\left(\frac{1}{\rho_{v}(x)^{p}}+(p-1)\left(\frac{2}{D_{\nu}(x)}\right)^{p}\right) d \omega(\nu)|u(x)|^{p} d x\right) .
\end{gathered}
$$

Now note that

$$
\int_{S^{n-1}}|\cos (\nu, \nabla u)|^{p} d \omega(\nu)=\int_{S^{n-1}}|\cos (e, \nu)|^{p} d \omega(\nu)
$$

for any fixed unit vector $e \in \mathbb{R}^{n}$. Elementary calculations show that

$$
\int_{S^{n-1}}|\cos (e, \nu)|^{p} d \omega(\nu)=\frac{\Gamma\left(\frac{p+1}{2}\right) \Gamma\left(\frac{n}{2}\right)}{\sqrt{\pi} \cdot \Gamma\left(\frac{n+p}{2}\right)} .
$$

By dividing both sides in (2.3) with the latter quantity and using the above lemma, we get

$$
\begin{aligned}
\int_{\Omega}|\nabla u|^{p} d x & \geq \frac{c_{p} \sqrt{\pi} \cdot \Gamma\left(\frac{n+p}{2}\right)}{\Gamma\left(\frac{p+1}{2}\right) \Gamma\left(\frac{n}{2}\right)}\left(\int_{\Omega} \int_{S^{n-1}} \frac{1}{\rho_{v}(x)^{p}} d \omega(\nu)|u(x)|^{p} d x\right. \\
& \left.+(p-1)\left(\frac{\left|\mathbb{S}^{n-1}\right|}{n}\right)^{\frac{p}{n}} \int_{\Omega} \frac{|u(x)|^{p}}{\left|\Omega_{x}\right|^{\frac{p}{n}}} d x\right),
\end{aligned}
$$

as desired. By standard density arguments, we get the same inequality for all real-valued $u \in \stackrel{\circ}{\mathrm{W}}^{1, p}(\Omega)$.

Now take an arbitrary $v(x) \in C_{0}^{\infty}(\Omega)$ (not nessesarily real-valued). Then we have $|v| \in \stackrel{\circ}{\mathrm{W}}^{1, p}(\Omega)$. Hence, we get the inequality (2.2) for the function $u(x)=|v(x)|$. Since $|\nabla| v(x)|| \leq|\nabla v(x)|$ a.e. (see, for example, E. H. Lieb and M. Loss [10], p. 144), we get inequality (2.2) for all $u \in C_{0}^{\infty}(\Omega)$ and thus for all $u \in \stackrel{\circ}{\mathrm{W}^{1, p}}(\Omega)$. This concludes the proof of the theorem.

For convex domains an easy geometric argument shows that

$$
\int_{S^{n-1}} \frac{1}{\rho_{\nu}(x)^{p}} d \omega(\nu) \geq \int_{S^{n-1}}|\cos (e, \nu)|^{p} d \omega(\nu) \cdot \frac{1}{\delta(x)^{p}}=\frac{\Gamma\left(\frac{p+1}{2}\right) \Gamma\left(\frac{n}{2}\right)}{\sqrt{\pi} \cdot \Gamma\left(\frac{n+p}{2}\right) \delta(x)^{p}} .
$$

For such domains we also know that $\Omega=\Omega_{x}$ for every $x \in \Omega$. Using the above theorem, we get

Theorem 2.2. For any convex domain $\Omega \subset \mathbb{R}^{n}$ and $u \in \stackrel{\circ}{\mathrm{W}}^{1, p}(\Omega)$ we have

$$
\int_{\Omega}|\nabla u|^{p} d x \geq c_{p} \int_{\Omega} \frac{|u(x)|^{p}}{\delta^{p}(x)} d x+\frac{a(p, n)}{|\Omega|^{\frac{p}{n}}} \int_{\Omega}|u(x)|^{p} d x,
$$

where

$$
a(p, n)=\frac{(p-1)^{p+1}}{p^{p}} \cdot\left(\frac{\left|\mathbb{S}^{n-1}\right|}{n}\right)^{\frac{p}{n}} \cdot \frac{\sqrt{\pi} \cdot \Gamma\left(\frac{n+p}{2}\right)}{\Gamma\left(\frac{p+1}{2}\right) \Gamma\left(\frac{n}{2}\right)} .
$$


If $\Omega$ is not convex, we have the following counterpart of Corollary 3.1 in 9 .

Corollary 2.1. Suppose there exists a constant $\kappa$ such that for each $y \in \partial \Omega$ and

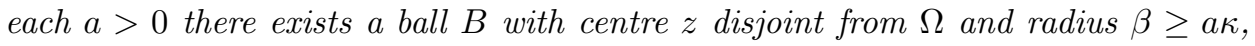
where $|z-y|=a$. Then there exists a constant $\theta \leq c_{p}$ such that

$$
\frac{c_{p} \cdot \sqrt{\pi} \cdot \Gamma\left(\frac{n+p}{2}\right)}{\Gamma\left(\frac{p+1}{2}\right) \Gamma\left(\frac{n}{2}\right)} \int_{S^{n-1}} \frac{1}{\rho_{\nu}(x)^{p}} d \omega(\nu) \geq \theta \frac{1}{\delta^{p}(x)}
$$

and hence

$$
\begin{aligned}
\int_{\Omega}|\nabla u|^{p} d x & \geq \theta \int_{\Omega} \int_{S^{n-1}} \frac{1}{\rho_{v}(x)^{p}} d \omega(\nu)|u(x)|^{p} d x \\
& +(p-1) \frac{c_{p} \sqrt{\pi} \cdot \Gamma\left(\frac{n+p}{2}\right)}{\Gamma\left(\frac{p+1}{2}\right) \Gamma\left(\frac{n}{2}\right)}\left(\frac{\left|\mathbb{S}^{n-1}\right|}{n}\right)^{\frac{p}{n}} \int_{\Omega} \frac{|u(x)|^{p}}{\left|\Omega_{x}\right|^{\frac{p}{n}}} d x .
\end{aligned}
$$

\section{ACKNowledgments}

I wish to thank my supervisor Ari Laptev for his help and for suggesting the problem.

\section{REFERENCES}

[1] G. Barbatis, S. Filippas, and A. Tertikas, A unified approach to improved $L^{p}$ Hardy inequalities with best constants, preprint, December 2000.

[2] H. Brezis and M. Marcus, Hardy's inequalities revisited, dedicated to Ennio De Giorgi, Ann. Scuola Norm. Sup. Pisa Cl. Sci. (4) vol. 25 (1997) no. 1-2 (1998), pp. 217-237. MR 99m: 46075

[3] E. B. Davies, A review of Hardy inequalities, Oper. Theory Adv. Appl., vol. 110 (1998), pp. 55-67. MR 2001f:35166

[4] E. B. Davies, Heat kernels and spectral theory, Cambridge University Press, Cambridge, 1989. MR 92a:35035

[5] E. B. Davies, The Hardy constant, Quart. J. Math. Oxford (2), vol. 46 (1995), pp. 417-431. MR 97b:46041

[6] E. B. Davies, Spectral theory and differential operators, Cambridge Studies in Advanced Mathematics, vol. 42, Cambridge University Press, Cambridge, 1995. MR 96h:47056

[7] G. Hardy, J. E. Littlewood, and G. Pólya, Inequalities, second edition, Cambridge Mathematical Library, Cambridge University Press, Cambridge, 1952. MR 13:727e

[8] G. Hardy, Note on a theorem of Hilbert, Math. Zeitschrift (6) (1920), pp. 314-317.

[9] M. Hoffmann-Ostenhof, T. Hoffmann-Ostenhof, and A. Laptev, A geometrical version of Hardy's inequality, J. Funct. Anal. 189 (2002), no. 2, 539-548. MR 2003c:26022

[10] E. H. Lieb and M. Loss, Analysis, Graduate Studies in Mathematics, vol. 14, American Mathematical Society, Providence, RI, 1997. MR 98b:00004

[11] T. Matskewich and P. E. Sobolevskii, The best possible constant in a generalized Hardy's inequality for convex domains in $\mathbb{R}^{n}$, Nonlinear Anal., vol. 28 (1997), pp. 1601-1610. MR 98a:26019

[12] V. G. Maz'ja, Sobolev Spaces, Springer-Verlag, Berlin, 1985. MR 87g:46056

[13] B. Opic and A. Kufner, Hardy-type inequalities, Pitman Research Notes in Mathematics Series, vol. 219, Longman Group UK Limited, London, 1990. MR 92b:26028

Department of Mathematics, University of Stockholm, 10691 Stockholm, Sweden

E-mail address: jespert@math.su.se 